Archive for

Organic Chemistry

Arkivoc 2018, part iv, 102-113

\title{
Synthesis of a chlorin with annelated lactam ring as subunit for artificial photosynthetic reaction centres
}

\author{
Franz-Peter Montforts*b and Elmar Haake ${ }^{a}$ \\ aStaas-und Universitätsbibliothek, University of Bremen, Bibliothekstr. 9, 28359 Bremen, Germany \\ bInstitute for Organic and Analytical Chemistry, FB2, University of Bremen, P.O. Box 330440, \\ 28334 Bremen, Germany \\ Email: mont@uni-bremen.de
}

\section{Dedicated to Prof. Gordon Gribble}

Received 12-31-2017

Accepted 02-12-2018

Published on line 03-04-2018

\section{Abstract}

A chlorin with an annelated cyclic ketone moiety was synthesized from a tricyclic nickel complex and appropriate pyrrole building blocks. Ketone functionality of the target chlorin allows Beckmann rearrangement to yield chlorin lactams. Lactam moieties on the chlorin represent masked amino acid structures which should allow formation of peptide-like backbones, along which chlorin pigments are arranged. Due to the "natural" chlorin chromophores and the peptide backbone the devices should represent artificial mimetics of natural photosynthesis systems.

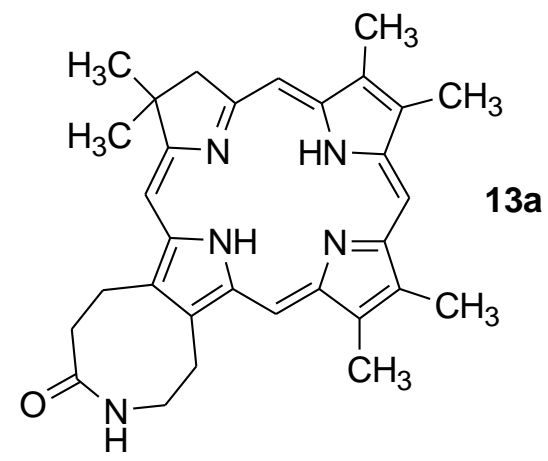

Keywords: Chlorin, pyrrole, Beckmann rearrangement, lactam, artificial photosynthesis 


\section{Introduction}

The elementary step of photosynthesis in bacteria, algae and, plants consists of a light-induced electron transfer from so-called special pair chlorophylls along a chain of further chlorophyll pigments to quinone acceptors. The so-formed hydroquinone structure provisionally stores two electrons for subsequent biochemical transformations. ${ }^{1-3}$ The process of light induced-electron transfer is accompanied by formation of proton gradients which are used for ATP production. Knowledge of the structure of photosynthetic reaction centres of bacteria and plants originates from crystal structure investigations. ${ }^{4-7}$ Crystal structure investigations revealed not only the spacial orientation but also that of the membrane protein environment in which the chlorophyll pigments are embedded by lipophilic interactions. For studying the complex photophysical/photochemical process of light-induced electron transfer in natural photosynthesis on a level of lower complexity, numerous artificial photosynthesis model systems were designed and synthesized. ${ }^{8-11}$ The majority of those model systems made use of porphyrin pigments; chlorin based systems are less widespread. ${ }^{12-16}$ Chlorin $\mathbf{2}$ the dihydroporphyrin chromophore of chlorophyll $a \mathbf{1}$ has different photophysical properties compared to the completely unsaturated porphyrins (Figure 1). The ubiquitous green colour of chlorophylls and the red of porphyrinoid blood pigment heme make this difference palpable. ${ }^{17-21}$

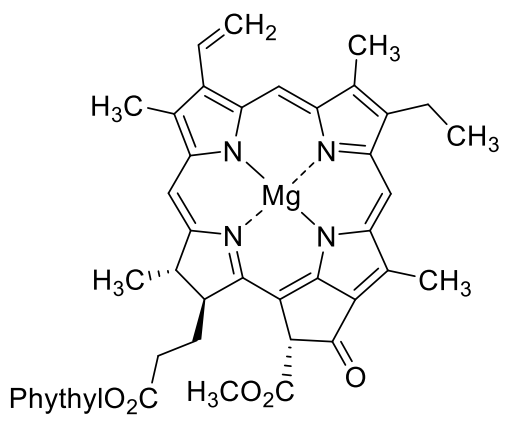

1

Chlorophyll a

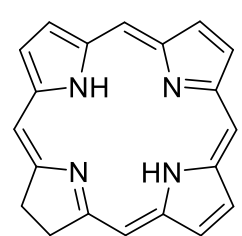

2

Chlorin

Figure 1. Chlorophyll $a \mathbf{1}$ and parent framework of chlorin 2.
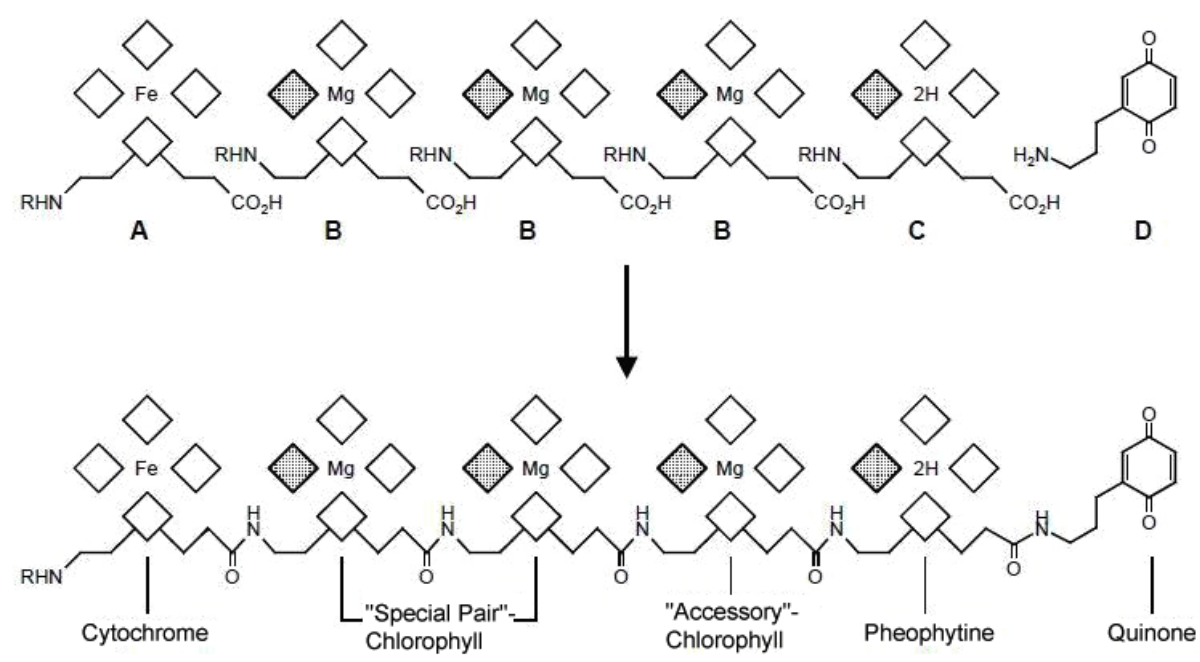

Figure 2. Schematic representation of chlorin subunits arranged along an artificial peptide backbone. 
To obtain more biomimetic photosynthesis models we aimed at the synthesis of chlorin type pigments with amino acid functionality. The chlorin subunits could then be covalently linked to peptide-like oligomers using established coupling methods from peptide chemistry (Figure 2).

Chlorin pigments arranged along the artificial peptide backbone could mimic the natural design in which chlorophylls are held together by lipophilic interactions with the protein environment.

\section{Results and Discussion}

The tricyclic nickel complex $\mathbf{9}$ (Scheme 2) which was used for several syntheses of chlorins in our laboratory is also an ideal intermediate for preparation of chlorins with the desired amino acid functionality. ${ }^{22-28}$ The task therefore was to connect 9 with pyrrole ring D building blocks which contain annuleted cycloketone moieties as masked amino acid functions.
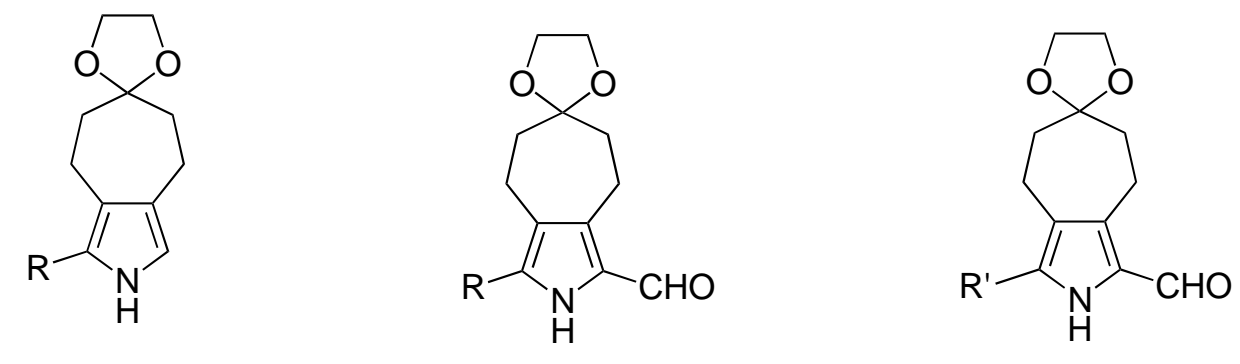

$$
\begin{aligned}
& 3 \mathrm{R}=\mathrm{CO}_{2} \mathrm{Bn} \\
& 6 \mathrm{R}=\mathrm{CN}
\end{aligned}
$$

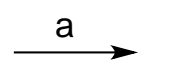

$4 \mathrm{R}=\mathrm{CO}_{2} \mathrm{Bn}$

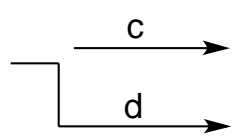

$5 \mathrm{R}^{\prime}=\mathrm{Br}$

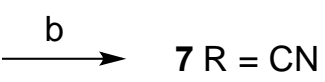

$8 R^{\prime}=1$

Scheme 1. Preparation of ring $D$ building blocks for chlorin synthesis. Reaction conditions. (a) $\mathrm{POCl}_{3}, \mathrm{DMF}$ $40{ }^{\circ} \mathrm{C}, 45 \mathrm{~min}$ (86\%). (b) $\mathrm{POCl}_{3}, \mathrm{DMF}, 40{ }^{\circ} \mathrm{C}, 16 \mathrm{~h}$ (61\%). (c)(i) $\mathrm{H}_{2}$ (1 atm), Pd/C, THF, NEt $, \mathrm{rt}, 30 \mathrm{~min}(100 \%)$; (ii) NBS, DMF, $50{ }^{\circ} \mathrm{C}, 1 \mathrm{~h}(44 \%)$. (d)(i) $\mathrm{H}_{2}$ (1 atm), Pd/C, THF, NEt $3, \mathrm{rt}, 30 \mathrm{~min}(100 \%)$; (ii) $\mathrm{NaHCO}_{3}, \mathrm{H}_{2} \mathrm{O}, \mathrm{I}_{2}, \mathrm{KI}$, $\mathrm{MeOH}, 70^{\circ} \mathrm{C}$, ca. $40 \mathrm{~min}(47 \%)$.

Pyrroles 3 and 6 were chosen as starting materials for ring D building blocks (Scheme 1). Preparation of 3 and 6 was achieved from cyclohept-4-ene-1-one and isocyanides according a general protocol for pyrrole synthesis developed in our laboratory. ${ }^{29,30}$

Condensation of ring $D$ building pyrroles with nickel tricycle 9 requires aldehyde functions. Therefore pyrrole benzyl ester 3 was subjected to Vilsmeier reaction conditions to yield $\mathbf{4}$. Debenzylation by catalytic hydrogenolysis and subsequent decarboxylative halogenations gave potential ring D building blocks $\mathbf{5}$ and $\mathbf{8}$. Bromide and iodide should function as leaving groups for the final cyclization step forming the chlorin. An alternative route started from cyano pyrrole 6 which yielded ring D building block directly by Vilsmeier formylation. The cyano group acts as leaving group for the final cyclization reaction.

With ring $D$ building blocks in hand, synthesis of chlorin 12 could be achieved (Scheme 2). The sequence started with hydrolysis of the ester group of nickel complex $\mathbf{9}$ and condensation with ring $D$ pyrrole aldehyde 7. Hydrolysis is facilitated by nickel complexation of the ethyl carboxylate group. Acid induced condensation proceeded with decarboxylation and decomplexation of nickel. The acidic reaction conditions also led to partial hydrolysis of the ketal function. 
<smiles></smiles>

9

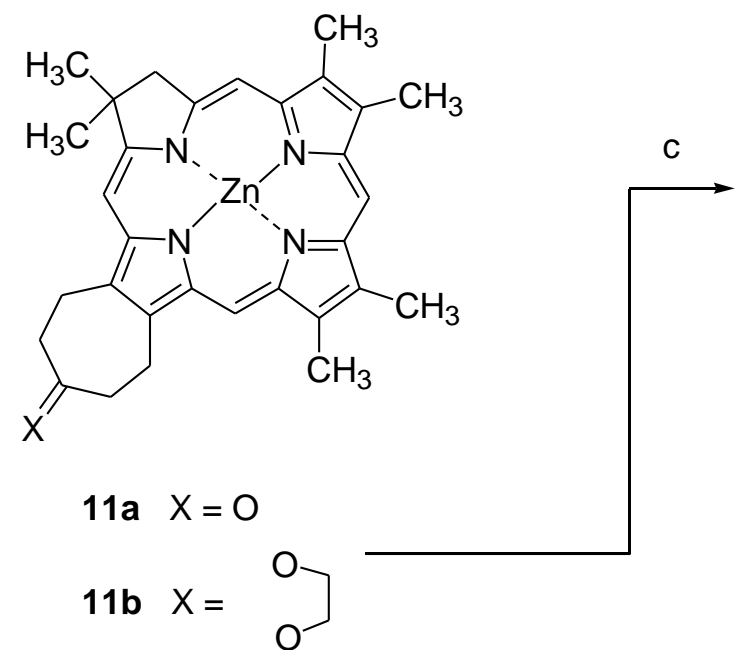<smiles>[X]C1CCC2=C(CC1)C(C)=NC2=Cc1[nH]c(/C=C2\N=C(/C=C3\CC(C)(C)C(C)(C)N3)C(C)=C2C)c(C)c1C</smiles>

10a $X=0$

10b $X=$

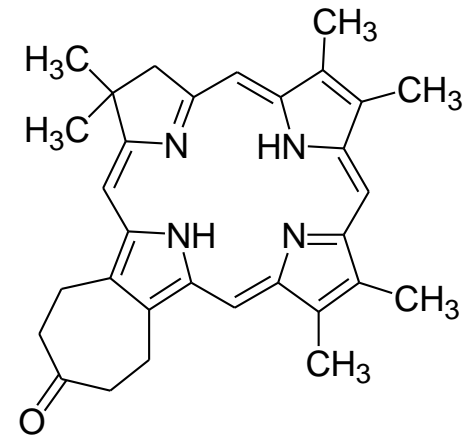

12

Scheme 2. Synthesis of chlorin 12. Reaction conditions. (a)(i) $5 \mathrm{~N} \mathrm{KOH}, \mathrm{MeOH} / \mathrm{H}_{2} \mathrm{O}(9: 1), \mathrm{THF}, 70{ }^{\circ} \mathrm{C}, 45 \mathrm{~min}$; (ii) + 7, $\mathrm{CHCl}_{3}, \mathrm{pTsOH}, 70^{\circ} \mathrm{C}, 10 \mathrm{~min}$ (72\% mixture of 10a, 10b). (b) $\mathrm{Zn}(\mathrm{OAc})_{2}, \mathrm{DBU}$, sulfolane, $145^{\circ} \mathrm{C}, 14 \mathrm{~h}$ (mixture of 11a,11b, 49\% related to 9). (c) $\mathrm{HClO}_{4}, \mathrm{MeCN}, \mathrm{H}_{2} \mathrm{O}, \mathrm{rt}, 15 \mathrm{~min}$ (97\%).

Thus tetracyclic bilin was formed in good yield as mixture of ketone $10 \mathrm{a}$ and ketal $\mathbf{1 0 b}$ derivatives. Because keto chlorin 12 was envisaged as the final target, we did not make any attempts to separate ketone and ketal derivatives on a preparative scale. To perform cyclization of bilin $\mathbf{1 0} \mathbf{a}, \mathbf{b}$ to chlorins $\mathbf{1 1} \mathbf{a}, \mathbf{b}$ the bilin was recomplexed with zinc(II) diacetate. The zinc stabilizes the quite sensitive bilin and, what is more important, it exercises an essential template effect for the cyclization process. ${ }^{22,23}$ Cyclization was initiated by base-induced elimination of HCN from the reduced pyrrole ring with formation of an enamine structure. The enamine attacks as a nucleophile at the cyano-substituted position of pyrrole ring $D$. Thus a methine bridge between rings $A$ and $B$ is formed with loss of a second $\mathrm{HCN}$ fragment. The obtained zinc chlorin $\mathbf{1 1 a , b}$ existed again as mixture of ketone 11a and ketal 11b derivatives.

Treatment of the mixture of chlorin derivatives $\mathbf{1 1 a , b}$ with perchloric acid removes the central zinc(II) and hydrolysed the ketal function to yield single keto chlorin 12 in good overall yield.

With iodo pyrrole aldehyde $\mathbf{8}$ as ring $\mathrm{D}$ building block only negligibly lower overall yields for the entire reaction sequence were achieved, but here reproducibility suffered due to the sensitivity of the bilin intermediates. With bromo pyrrole aldehyde $\mathbf{5}$, synthesis of bilins/chlorins failed completely. 
Using keto chlorin 12 a Beckmann rearrangement for formation of lactam targets was performed (Scheme 3).

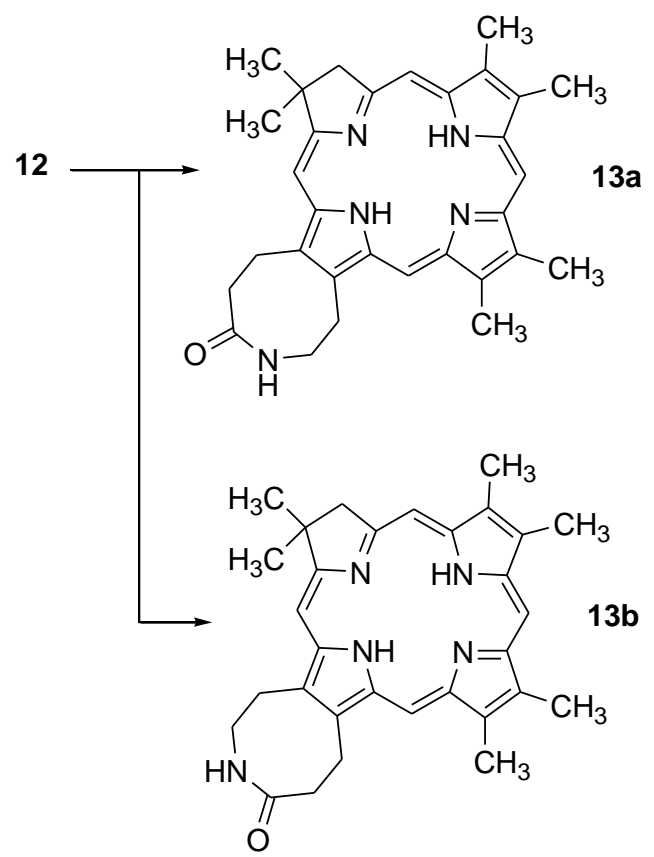

Scheme 3. Formation of chlorin lactams. Reaction conditions. (a)(i) O-mesityl sulfonyl hydroxylamine, $\mathrm{CH}_{2} \mathrm{Cl}_{2}, 0^{\circ} \mathrm{C}$ to rt; (ii) $\mathrm{Al}_{2} \mathrm{O}_{3}$ basic, activity I, in $\mathrm{MeOH}$, benzene, rt, $3 \mathrm{~h}$ (77\% mixture of 13a, 13b).

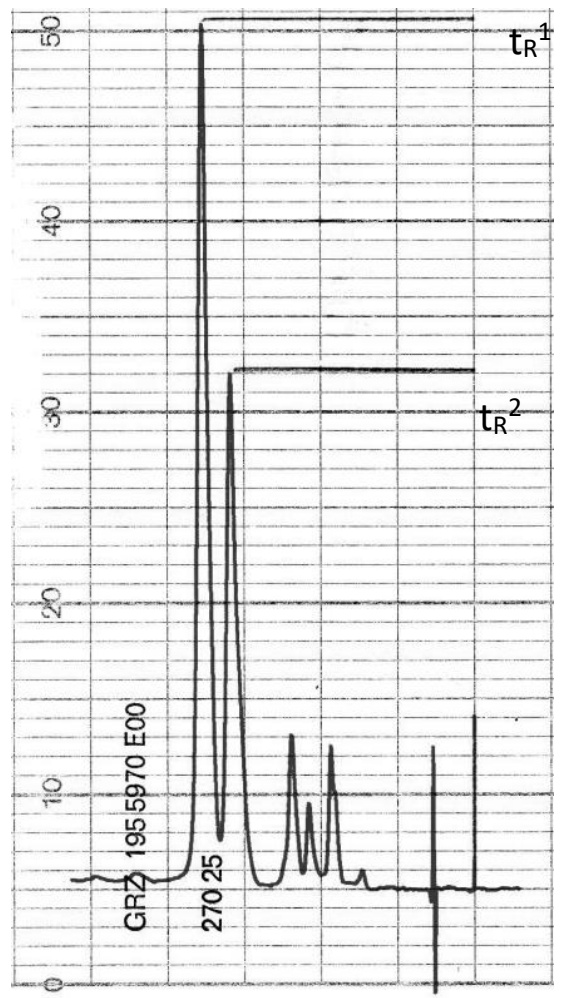

Figure 3. HPLC of reaction mixture from the Beckmann rearrangement indicating the ratio of formed lactams $13 b\left(t_{R}{ }^{2}=16 \mathrm{~min}\right)$ and $13 a\left(t_{R}{ }^{1}=17.5 \mathrm{~min}\right)$. Separation conditions: Nucleosil Chiral 2, $n$ heptane/dioxane 7:3,1 $\mathrm{mL} / \mathrm{min}$, UV detection at $405 \mathrm{~nm}$. 
O-Mesityl sulfonyl hydroxylamine (Tamura reagent) was reported as a reagent that allows very gentle reaction conditions (temperatures around $0{ }^{\circ} \mathrm{C}$ ) for Beckmann rearrangements. ${ }^{31,32}$ Another advantage of this hydroxylamine derivative could be its sterical strain with possible preferred formation of one constitutional lactam isomer. Indeed, the rearrangement reaction of keto chlorin $\mathbf{1 2}$ could be achieved with good yields under very gentle reaction conditions.

Analytical HPLC and ${ }^{1} \mathrm{H}$ NMR spectroscopy revealed that a mixture of constitutionally isomeric lactams $13 a$ and $\mathbf{1 3 b}$ was formed. Though constitutional isomer $\mathbf{1 3 a}$ was slightly preferred (13a:13b $=1.5: 1)$, the observed selectivity cannot be attributed to sterical strain of the Tamura reagent. Possible less favorable orientation of the mesityl residue in the oxime intermediate towards ring $A$ with bulky geminal dimethyl substitution should favor constitutional isomer $\mathbf{1 3 b}$ and not the observed isomer 13a. The constitutions of $\mathbf{1 3 a}$ and $\mathbf{1 3 b}$ were tentatively assigned by 2D-NOESY- and 2D-NOESY-HH-COSY experiments. Assignment was facilitated by the fact that the isomers have different proportions in the mixture.

\section{Conclusions}

Condensation of tricyclic nickel complex $\mathbf{9}$ and cyanopyrrole aldehyde $\mathbf{7}$ provided a facile synthetic access to ketochlorin 12. Subsequent cyclization of bilin intermediates $\mathbf{1 0}$ formed the macrocyclic chlorin in a $48 \%$ yield. The desired lactams were obtained by Beckmann rearrangement, also in good yields. Lactams $13 \mathbf{a}, \mathbf{b}$ as protected amino acid like subunits should open an access to artificial photosynthesis models with a peptide/polyamide backbone.

\section{Experimental Section}

General. Starting materials were prepared either according to literature procedures or were purchased from Fluka, Merck, Acros Organics or Sigma Aldrich and used without further purification. All solvents were purified and dried by standard methods. All reactions were carried out under argon. Melting points are not corrected. TLC: Silica gel plates (Riedel de Haën, silica gel 60 F 254; Macherey-Nagel, Polygram SIL G/UV 254) and aluminium oxide plates (Macherey \& Nagel, Polygram Alox N/UV 254.). Column chromatographic separations were performed on silica gel (ICN Biomedicals, 32-63 $\mu \mathrm{m}, 60 \AA ̊$ ). HPLC: Knauer HPLC instrument with pump 64, two-channel potentiometer BBC Metrawatt Servogor 120 recorder and Knauer UV spectrometer. UV/Vis: Kontron UVIKON 810 spectrophotometer and Perkin Elmer UV/Vis spectrophotometer Lambda 2. IR: PerkinElmer Paragon 500 FT-IR-spectrometer. NMR spectra: Bruker DPX-200 AVANCE, Bruker AM 360 spectrometer and Bruker AMX spectrometer. All chemical shifts were referenced to TMS lock signal. Exact assignment of proton signals in ${ }^{1} \mathrm{H}$ NMR spectra was achieved by two dimensional $\mathrm{H}, \mathrm{H}-\mathrm{COSY}$ and NOESY experiments. MS: Finnigan MAT 8200 and CH7A MAT spectrometer [E (70 eV) and DCl ( $\left.\mathrm{NH}_{3}, 8 \mathrm{~mA} / \mathrm{s}\right)$ ]. HRMS: Finnigan MAT 8200 spectrometer according peak matching method. Elemental Analysis: Microanalytical Laboratories Beller, Göttingen, Germany and Pascher, Remagen, Germany.

Benzyl 3-formyl-4,5,7,8-tetrahydrospiro-[cyclohepta[c]pyrrole-6(2H),2'-[1,3]dioxolane]-1-carboxylate (4). To a solution of benzyl 4,5,7,8-tetrahydrospiro-[cyclohepta[c]pyrrole-6(2H),2'-[1,3]dioxolane]-1-carboxylate (3) $(394 \mathrm{mg}, 1.2 \mathrm{mmol}$ ) in DMF $(8 \mathrm{~mL})$ was added with stirring under an argon atmosphere a solution of Vilsmeier reagent $(440 \mu \mathrm{L})$ at $0{ }^{\circ} \mathrm{C}$. The Vilsmeier reagent was separately prepared from $\mathrm{POCl}_{3}(330 \mu \mathrm{L}, 554 \mathrm{mg}$, 
$3.6 \mathrm{mmol}$ ) and DMF (560 $\mu \mathrm{L})$ under an argon atmosphere. After being stirred for $45 \mathrm{~min}$ at $40{ }^{\circ} \mathrm{C}$, aqueous saturated NaOAc $(20 \mathrm{~mL})$ was added and stirring was continued for $15 \mathrm{~min}$. After being cooled to $\mathrm{rt}$, the reaction mixture was poured into a separating funnel which contained ice water $(30 \mathrm{~mL})$ and it was extracted five times with $\mathrm{CH}_{2} \mathrm{Cl}_{2}$ (20 $\mathrm{mL}$ each portion). The combined organic layers were dried by filtration through cotton wool and evaporated. The colorless solid was purified through flash chromatography [silica gel (40 g), $\mathrm{CH}_{2} \mathrm{Cl}_{2}$ /EtOAc 9:1]. After removal of the eluent and crystallization from $\mathrm{CHCl}_{3} / n$-pentane 4 was obtained as colorless crystals (366 mg, 86\%). mp $101{ }^{\circ} \mathrm{C}$. TLC(silica gel, $\mathrm{CH}_{2} \mathrm{Cl}_{2} /$ EtOAc 9:1): $\mathrm{R}_{\mathrm{f}}$ 0.54. IR (solid, $\mathrm{KBr}$, vmax, $\left.\mathrm{cm}^{-1}\right)$ : 3298s (N-H), 2949m (CH, aliph), 1705s (C=O), 1660s (C=O), 1560, 1464, 1253, 1192, 1109, 1282, 1032, 983, 947. ${ }^{1} \mathrm{H} N M R\left(360 \mathrm{MHz}, \mathrm{CDCl}_{3}\right): \delta_{\mathrm{H}} 2.84\left[4 \mathrm{H}, \mathrm{m}, \mathrm{H}_{2} \mathrm{C}(5), \mathrm{H}_{2} \mathrm{C}(7)\right], 2.91\left[2 \mathrm{H}, \mathrm{m}, \mathrm{H}_{2} \mathrm{C}(4)\right], 3.07[2 \mathrm{H}$, $\left.\mathrm{m}, \mathrm{H}_{2} \mathrm{C}(8)\right], 4.02\left[4 \mathrm{H}, \mathrm{m}, \mathrm{H}_{2} \mathrm{C}\left(4^{\prime \prime}\right), \mathrm{H}_{2} \mathrm{C}\left(5^{\prime \prime}\right)\right], 5.32\left(2 \mathrm{H}, \mathrm{m}, \mathrm{CH}_{2} \mathrm{Ph}\right), 7.40\left(5 \mathrm{H}, \mathrm{m}, \mathrm{C}_{6} \mathrm{H}_{5}\right), 9.37$ (1H, broad s, $\mathrm{NH}), 9.77(1 \mathrm{H}, \mathrm{s}, \mathrm{CHO}) . \mathrm{El}-\mathrm{MS}\left(70 \mathrm{eV}, 20{ }^{\circ} \mathrm{C}\right): \mathrm{m} / z(\%) 355\left(66, \mathrm{M}^{+}\right), 264\left(100,\left[\mathrm{M}-\mathrm{C}_{7} \mathrm{H}_{7}\right]^{+}\right), 246(22), 174$ (12), 91 (96, $\left[\mathrm{C}_{7} \mathrm{H}_{7}\right]^{+}$. Anal. calcd for $\mathrm{C}_{20} \mathrm{H}_{21} \mathrm{NO}_{5}$ (355.39): C, 67.59; $\mathrm{H}, 5.97 ; \mathrm{N}, 3.94$. Found: $\mathrm{C}, 67.69 ; \mathrm{H}, 6.04$; N, 3.87.

3-Bromo-4,5,7,8-tetrahydrospiro[cyclohepta[c]pyrrole-6(2H),2'-[1,3]dioxolane]-1-carbaldehyde (5). To a solution of formyl benzyl ester 2 (80 mg, $225 \mu \mathrm{mol})$ in THF $(15 \mathrm{~mL})$ were added few drops of $\mathrm{NEt}_{3}$ and a small amount of $\mathrm{Pd} / \mathrm{C}$ catalyst under an argon atmosphere. After replacement of argon by hydrogen the mixture was hydrogenated under stirring for ca. $30 \mathrm{~min}$. The catalyst was removed by filtration through Celite 521 , washed with THF and, the solvent evaporated. After removal of $\mathrm{NEt}_{3}$ in vacuo of an oil pump, 3-formyl4,5,7,8-tetrahydrospiro[cyclohepta[c]pyrrole-6(2H),2'-[1,3]dioxolane]-1-carboxylic acid (4a) was obtained as a colorless solid (60 mg, 100\%). TLC (silica gel, $\left.\mathrm{CH}_{2} \mathrm{Cl}_{2} / \mathrm{EtOAc} 9: 1\right): \mathrm{R}_{\mathrm{f}}$ 0.1. El-MS (70 eV, $\left.200{ }^{\circ} \mathrm{C}\right): \mathrm{m} / z(\%)$ $265\left(69, \mathrm{M}^{+}\right), 221\left(8,\left[\mathrm{M}-\mathrm{C}_{2} \mathrm{H}_{4} \mathrm{O}\right]^{+}\right), 86(100), 44\left(10, \mathrm{C}_{2} \mathrm{H}_{4} \mathrm{O}^{+}\right)$.

The carboxylic acid was used for the next reaction step without complete characterization.

To a solution of pyrrole carboxylic acid $4 a(60 \mathrm{mg}, 225 \mu \mathrm{mol})$ in DMF (2 mL) was added slowly $N$-bromo succinimide $(80 \mathrm{mg}, 450 \mu \mathrm{mol})$ dissolved in DMF $(2 \mathrm{~mL})$ and the mixture was stirred for $1 \mathrm{~h}$ at $50{ }^{\circ} \mathrm{C}$. After being cooled to $\mathrm{rt}$, the reaction mixture was poured into a separating funnel which contained water (10 $\mathrm{mL})$ and extracted three times with $\mathrm{CH}_{2} \mathrm{Cl}_{2}$ (10 $\mathrm{mL}$ each portion). The combined organic extracts were dried by filtration through cotton wool and evaporated. The residue was purified by flash chromatography [silica gel (20 g), $\mathrm{CH}_{2} \mathrm{Cl}_{2} /$ EtOAc 9:1]. After removal of the eluent a light-yellow oil was obtained which was crystallized from $\mathrm{CHCl}_{3} / n$-pentane to yield light pink crystals of $5(30.4 \mathrm{mg}, 45 \%)$ mp $172-173{ }^{\circ} \mathrm{C}$. TLC (silica gel, $\mathrm{CH}_{2} \mathrm{Cl}_{2}$ /EtOAc 9:1): $\mathrm{R}_{\mathrm{f}}$ 0.21. IR (solid, $\mathrm{KBr}, \mathrm{vmax}, \mathrm{cm}^{-1}$ ): 3222s (N-H), 3015, 2937, 2880, 1639s (C=O), 1428, 1377, $1339,1278,1200,1110,1068,1034,984,948,877,816,743 .{ }^{1} \mathrm{H} N M R\left(360 \mathrm{MHz}, \mathrm{CDCl}_{3}\right): \delta_{\mathrm{H}} 1.80[2 \mathrm{H}, \mathrm{m}$, $\left.\mathrm{H}_{2} \mathrm{C}(5)\right], 1.87\left[2 \mathrm{H}, \mathrm{m}, \mathrm{H}_{2} \mathrm{C}(7)\right], 2.57\left(2 \mathrm{H}, \mathrm{m}, \mathrm{H}_{2} \mathrm{C}(4)\right], 2.9\left[2 \mathrm{H}, \mathrm{m}, \mathrm{H}_{2} \mathrm{C}(8)\right], 4.01\left[4 \mathrm{H}, \mathrm{s}, \mathrm{H}_{2} \mathrm{C}\left(4^{\prime}\right), \mathrm{H}_{2} \mathrm{C}\left(5^{\prime}\right)\right], 9.12$ $(1 \mathrm{H}$,broad s, NH), $9.49(1 \mathrm{H}, \mathrm{s}, \mathrm{CHO})$. Exact attribution of proton signals was determined by 1D-HH-SEL-NOESYand 1D-HH-SEL-COSY-experiments. EI-MS (70 eV, $\left.200{ }^{\circ} \mathrm{C}\right): m / z(\%) 301\left(100,\left[M,{ }^{81} \mathrm{Br}\right]^{+}\right), 299\left(97,\left[M,{ }^{79} \mathrm{Br}\right]^{+}\right)$, $258\left(12,\left[M,{ }^{81} \mathrm{Br}\right]^{+}-\mathrm{C}_{2} \mathrm{H}_{3} \mathrm{O}\right), 256\left(31,\left[\mathrm{M},{ }^{79} \mathrm{Br}\right]^{+}-\mathrm{C}_{2} \mathrm{H}_{3} \mathrm{O}\right), 220\left(36,[\mathrm{M}-\mathrm{Br}]^{+}\right), 213(19), 186(6), 148(7), 118(5), 105$ (10), 87 (13), 65 (11), 43 (17). HRMS: Calcd for $\left(\mathrm{C}_{12} \mathrm{H}_{14} \mathrm{NO}_{3}{ }^{79} \mathrm{Br}^{+}\right)$299.01572. Found: 299.015.

3-lodo-4,5,7,8-tetrahydrospiro[cyclohepta[c]pyrrole-6(2H),2'-[1,3]dioxolane]-1-carbaldehyde (8). Pyrrole carboxylic acid (4a) (143 mg, $0.54 \mathrm{mmol}$ ) was prepared by hydrogenolysis as described above and mixed together with $\mathrm{NaHCO}_{3}(181 \mathrm{mg}, 2.16 \mathrm{mmol})$ in water $(20 \mathrm{~mL})$. The mixture was heated under an argon atmosphere to $70{ }^{\circ} \mathrm{C}$ until the solution became homogenous. At the same time $\mathrm{KI}(266 \mathrm{mg}, 1.6 \mathrm{mmol})$ and $\mathrm{I}_{2}$ $(150 \mathrm{mg}, 670 \mu \mathrm{mol})$ were dissolved in $\mathrm{MeOH}(6 \mathrm{~mL})$ by treatment in an ultrasonic bath. The iodine solution was then added during $30 \mathrm{~min}$ at $70{ }^{\circ} \mathrm{C}$ to the solution of carboxylic acid. After stirring for additional $10 \mathrm{~min}$ at $70{ }^{\circ} \mathrm{C}, \mathrm{NaS}_{2} \mathrm{O}_{3}$ was added and the reaction mixture was cooled to rt. The aqueous layer was extracted four times with $\mathrm{CH}_{2} \mathrm{Cl}_{2}$ (10 $\mathrm{mL}$ each portion). The combined organic extracts were dried by filtration through cotton 
wool, the solvent evaporated and the residue dried in vacuo of an oil pump. The residue was purified by flash chromatography [silica gel (30 g), $\mathrm{CH}_{2} \mathrm{Cl}_{2}$ /EtOAc 9:1]. After removal of the eluent the obtained solid was crystallized from $\mathrm{CHCl}_{3} / n$-pentane to yield brownish crystals (90 mg, 47\%). mp $189-190{ }^{\circ} \mathrm{C}$. TLC (silica gel, $\mathrm{CH}_{2} \mathrm{Cl}_{2}$ /EtOAc 9:1): $\mathrm{R}_{\mathrm{f}}$ 0.3. IR (solid, $\mathrm{KBr}, \mathrm{vmax}, \mathrm{cm}^{-1}$ ): 3217, 2948, 2877, 1644s (C=O), 1438, 1422, 1375, 1195, $1111,1068,1032,745) .{ }^{1} \mathrm{H}$ NMR $\left(360 \mathrm{MHz}, \mathrm{CDCl}_{3}\right): \delta_{\mathrm{H}} 1.79\left[2 \mathrm{H}, \mathrm{m}, \mathrm{H}_{2} \mathrm{C}(5)\right], 1.87\left[2 \mathrm{H}, \mathrm{m}, \mathrm{H}_{2} \mathrm{C}(7)\right], 2.54[2 \mathrm{H}, \mathrm{m}$, $\left.\mathrm{H}_{2} \mathrm{C}(4)\right], 2.91\left[2 \mathrm{H}, \mathrm{m}, \mathrm{H}_{2} \mathrm{C}(8)\right], 4.02$ [4H, m, $\left.\mathrm{H}_{2} \mathrm{C}\left(4^{\prime \prime}\right), \mathrm{H}_{2} \mathrm{C}\left(5^{\prime \prime}\right)\right], 8.93$ (1H, broad s, N-H), 9.40 (1H, s, CHO). El-MS $\left(70 \mathrm{eV}, 200{ }^{\circ} \mathrm{C}\right): \mathrm{m} / \mathrm{z}(\%) 347\left(91, \mathrm{M}^{+}\right), 304\left(20,\left[\mathrm{M}-\mathrm{C}_{2} \mathrm{H}_{3} \mathrm{O}\right]^{+}\right), 285$ (19), $274\left(20,\left[\mathrm{M}-\mathrm{C}_{2} \mathrm{H}_{4} \mathrm{O}-\mathrm{CHO}^{+}\right), 261(30)\right.$, 260 (23), $233(11), 220\left(46,[\mathrm{M}-\mathrm{I}]^{+}\right), 176\left(22,\left[\mathrm{M}-\mathrm{C}_{2} \mathrm{H}_{4} \mathrm{O}-\mathrm{I}\right]^{+}\right), 148(21), 147(21), 134$ (17), 130 (13), 120 (15, [M$\left.\left.\mathrm{C}_{7} \mathrm{H}_{12} \mathrm{O}_{2}\right]^{+}\right), 119$ (12), $118(22), 106$ (35), 105 (45), 104 (24), 103 (11), 93 (10), 91 (14), 87 (100), 79 (23), 77 (23), 45 (10), 43 (11). HRMS: Calcd for $\left(\mathrm{C}_{12} \mathrm{H}_{14} \mathrm{INO}_{3}{ }^{+}\right) 347.00183$. Found 347.00173

3-Formyl-4,5,7,8-tetrahydrospiro[cyclohepta[c]pyrrole-6(2H),2'-[1,3]dioxolane]-1-carbonitrile (7). To a solution of 4,5,7,8-tetrahydrospiro[cyclohepta[c]pyrrol-6(2H),2'-[1,3]dioxolan]-1-carbonitrile (6) ( $94 \mathrm{mg}, 431$ $\mu \mathrm{mol})$ in DMF $(10 \mathrm{~mL})$ was added with stirring under an argon atmosphere a solution of Vilsmeier reagent $(163 \mu \mathrm{mol})$ at $5{ }^{\circ} \mathrm{C}$. The Vilsmeier reagent was separately prepared from $\mathrm{POCl}_{3}(120 \mu \mathrm{L}, 202 \mathrm{mg}, 1.31 \mathrm{mmol})$ and DMF ( $210 \mu \mathrm{L}, 2.62 \mathrm{mmol})$ with stirring for $15 \mathrm{~min}$ at $15{ }^{\circ} \mathrm{C}$ under an argon atmosphere. After being stirred for $16 \mathrm{~h}$ at $40{ }^{\circ} \mathrm{C}$, aqueous saturated NaOAc $(25 \mathrm{~mL})$ was added and stirring was continued for $15 \mathrm{~min}$. After being cooled to rt the reaction mixture was poured into a separating funnel which contained ice water $(40 \mathrm{~mL})$ and extracted three times with $\mathrm{CH}_{2} \mathrm{Cl}_{2}$ (15 $\mathrm{mL}$ each portion). The combined organic layers were dried by filtration through cotton wool and evaporated. DMF residues were removed in vacuo of an oil pump and the obtained solid was purified by flash chromatography [silica gel $(20 \mathrm{~g}), \mathrm{CH}_{2} \mathrm{Cl}_{2} / \mathrm{EtOAc} 9: 1$ ]. After removal of eluent the residue was crystallized from $\mathrm{CHCl}_{3} / n$-pentane to yield colorless crystals (65 $\mathrm{mg}, 61 \%$ ). $\mathrm{mp} 180{ }^{\circ} \mathrm{C}$. TIC (silica gel, $\mathrm{CH}_{2} \mathrm{Cl}_{2}$ /EtOAc 9:1): $\mathrm{R}_{\mathrm{f}}$ 0.31. IR (solid, $\mathrm{KBr}, \mathrm{vmax}, \mathrm{cm}^{-1}$ ): 3259, 3026, 2928, 2887, 2855, 2728, 2222s (CN), 1653s (CHO), 1464, 1386, 1203, 1115, 1085, 1029, 949, 876, 825.

${ }^{1} \mathrm{H} N M R\left(360 \mathrm{MHz}, \mathrm{CDCl}_{3}\right): \delta_{\mathrm{H}} 1.89[2 \mathrm{H}, \mathrm{m}, \mathrm{H} 2 \mathrm{C}(5)], 1.95\left[2 \mathrm{H}, \mathrm{m}, \mathrm{H}_{2} \mathrm{C}(7)\right], 2.81[2 \mathrm{H}, \mathrm{m}, \mathrm{H} 2 \mathrm{C}(8)], 2.98[2 \mathrm{H}, \mathrm{m}$, $\left.\mathrm{H}_{2} \mathrm{C}(4)\right], 4.09\left[4 \mathrm{H}, \mathrm{m}, \mathrm{H}_{2} \mathrm{C}\left(4^{\prime}\right), 2 \mathrm{H}_{2} \mathrm{C}\left(5^{\prime}\right)\right], 9.34(1 \mathrm{H}$, broad $\mathrm{s}, \mathrm{N}-\mathrm{H}), 9.81(1 \mathrm{H}, \mathrm{s}, \mathrm{CHO})$. Exact attribution of proton signals was determined by $1 \mathrm{D}-\mathrm{HH}-\mathrm{SEL}-\mathrm{NOESY}$-experiments. El-MS $\left(70 \mathrm{eV}, 200{ }^{\circ} \mathrm{C}\right): \mathrm{m} / z(\%) 247\left(14,\left[\mathrm{M},{ }^{13} \mathrm{C}\right]^{+}\right)$, $246\left(100, M^{+}\right), 216(6), 203\left(11,\left[M+1-C_{2} \mathrm{H}_{4} \mathrm{O}\right]^{+}\right), 202\left(6,\left[M-\mathrm{C}_{2} \mathrm{H}_{4} \mathrm{O}\right]^{+}\right), 201(11), 184(8), 175$ (5), $174(19), 173$ (34), 172 (14), 171 (5), 160 (21), 159 (31), 157 (8), 155 (5), 147 (7), 146 (15), 145 (16), 143 (10), 142 (6), 133 (8), 132 (24), 131 (48), 130 (6), 129 (9), 119 (5), $118(10), 117$ (7), $116(8), 105(17), 104(23), 103$ (8), 102 (7), 99 (5), 97 (7), 91 (12), 90 (6), 89 (6), 88 (5), 87 (61), 86 (7), $86(14), 84(20), 83(6), 78$ (8), 77 (21), $76(6), 73(11), 71$ (10), 69 (10), 67 (6), 65 (12), 64 (7), 63 (6), 57 (18), 56 (6), 55 (19), 53 (9), 52 (7), 51 (9), 51 (11), 49 (33), 47 (5), 46 (15), 44 (18), 44 (20), 43 (20), 42 (18), 40 (15), 29 (8), 29 (5), 27 (12), 17 (17). HRMS: Calcd for $\left(\mathrm{C}_{13} \mathrm{H}_{14} \mathrm{~N}_{2} \mathrm{O}_{3}{ }^{+}\right)$ 246.10045. Found 246.10070. Anal. calcd for $\mathrm{C}_{13} \mathrm{H}_{14} \mathrm{~N}_{2} \mathrm{O}_{3}(248,66)$ : C, 63.40; $\mathrm{H}, 5.73 ; \mathrm{N}, 11.38$. Found C, 64.23; H 6.20; N 10.66 .

\section{0,21,22,23-Tetrahydro-10,11,15,16,21,21,22-heptamethyl-3-oxo-24H-cyclohepta[b]bilin-6,22-dicarbonitrile}

(10a) and its [1,3]-dioxolane derivative (10b). To [Ethyl-(14RS)-(14-cyano-12,13,14,17-tetrahydro2,3,8,13,13,14-heptamethyl-15H-tripyrrin-1-carboxylato)]nickel(II) (9) (5 mg, $10.5 \mu \mathrm{mol})$ in THF (2 mL) was added under stirring a $5 \mathrm{~N}$ solution of $\mathrm{KOH}$ in $\mathrm{MeOH} / \mathrm{H}_{2} \mathrm{O}(9: 1)(0.9 \mathrm{~mL}, 2.8 \mathrm{mmol})$ and the mixture was heated at $70{ }^{\circ} \mathrm{C}$ under an argon atmosphere for $45 \mathrm{~min}$. After being cooled to $\mathrm{rt}$, the mixture was poured with $\mathrm{CH}_{2} \mathrm{Cl}_{2}$ $\left(10 \mathrm{~mL}\right.$ ) into a separating funnel which contained a saturated aqueous $\mathrm{NaHCO}_{3}$ solution $(50 \mathrm{~mL})$. The aqueous layer was sufficiently extracted with $\mathrm{CH}_{2} \mathrm{Cl}_{2}$, the combined organic extracts were dried by filtration through cotton wool and evaporated in vacuo. To the obtained crude carboxylic acid of $\mathbf{9}$ was added under an argon atmosphere a solution of cyanopyrrole carbaldehyde 7 (3.9 $\mathrm{mg}, 16 \mu \mathrm{mol})$ in $\mathrm{CHCl}_{3}(2 \mathrm{~mL})$. To this solution was added via a syringe a $0.4 \mathrm{~N}$ degassed solution of dry $p$ - $\mathrm{TsOH}$ in $\mathrm{CHCl}_{3}(0.21 \mathrm{~mL}, 84 \mu \mathrm{mol})$. After being refluxed 
for 10 min and cooled to $\mathrm{rt}$, the reaction mixture was poured with $\mathrm{CH}_{2} \mathrm{Cl}_{2}(10 \mathrm{~mL})$ into a separating funnel which contained a saturated aqueous $\mathrm{NaHCO}_{3}$ solution $(50 \mathrm{~mL})$. The aqueous layer was extracted five times with $\mathrm{CH}_{2} \mathrm{Cl}_{2}$ (10 $\mathrm{mL}$ each portion) and the combined organic extracts were dried by filtration through cotton wool and evaporated in vacuo. The residue was purified by chromatography [Alox N (30 g), activity II-III, $\mathrm{CH}_{2} \mathrm{Cl}_{2} / \mathrm{MeOH}$ 97:3] to yield a mixture of deep blue bilins $10 \mathrm{a}, \mathbf{b}(4.4 \mathrm{mg} 72 \%$, yield is calculated for ketal $10 \mathbf{b}$ which predominates in the mixture). Due to instability of bilins the crude mixture was used for the next synthesis step without an extensive characterization. TLC (silica gel, $\mathrm{CH}_{2} \mathrm{Cl}_{2} / \mathrm{EtOAC}$ 9:1): $\mathrm{R}_{\mathrm{f}}{ }^{1} 0.72$ 10b; $\mathrm{R}_{\mathrm{f}}{ }^{2} 0,62$ 10a. UV/Vis $\left[\mathrm{CHCl}_{3}, \lambda, \mathrm{nm}\right.$, (relative intensities)]: 370 (100), 570 (45), 608 (46). DCl-MS (negative, $\left.\mathrm{NH}_{3} / \mathrm{NH}_{4}{ }^{+}, \mathrm{mA} / \mathrm{sec}\right): \mathrm{m} / z$ (\%) $576\left(12,\left[\mathrm{C}_{34} \mathrm{H}_{40} \mathrm{~N}_{6} \mathrm{O}\right]^{-}\right), 533\left(15,\left[\mathrm{C}_{32}{ }^{13} \mathrm{CH}_{36} \mathrm{~N}_{6} \mathrm{O}\right]^{-}\right), 532\left(100,\left[\mathrm{C}_{33} \mathrm{H}_{37} \mathrm{~N}_{5} \mathrm{O}\right]^{-}\right)$, $507\left(12,\left[\mathrm{C}_{32} \mathrm{H}_{37} \mathrm{~N}_{5} \mathrm{O}\right]^{-}\right)$.

\{9,10-Dihydro-9,9,14,15,19,20-hexamethyl-3-oxo-24H,26H-cyclohepta[b]porphyrinato\}zinc(II) (11a) and its [1,3]-dioxolane derivative (11b) . Bilin mixture 11a,b $(4.4 \mathrm{mg}, 7.6 \mu \mathrm{mol})$ was transferred with $\mathrm{CH}_{2} \mathrm{Cl}_{2}(\mathrm{ca} .2 \mathrm{~mL})$ into a glass ampule. After $\mathrm{CH}_{2} \mathrm{Cl}_{2}$ was removed in a stream of argon, $\mathrm{Zn}(\mathrm{OAc})_{2}(12 \mathrm{mg}, 52.5 \mu \mathrm{mol}, 7$ equiv.), sulfolane (1 mL) and 1,8-diazabicyclo[5.4.0]-undec-7-ene (DBU) (452 $\mu \mathrm{L}, 3.02 \mathrm{mmol})$ were added. The mixture was carefully degassed in vacuo of an oil pump and the ampule sealed by melting off. The reaction mixture was heated at $145^{\circ} \mathrm{C}$ for $14 \mathrm{~h}$. After being cooled to $\mathrm{rt}$, the ampule was cautiously opened and, the mixture was poured with $\mathrm{CH}_{2} \mathrm{Cl}_{2}(10 \mathrm{~mL})$ into a separating funnel. The organic layer was washed three times with brine (15 $\mathrm{mL}$ each portion). The combined brine layers were re-extracted with $\mathrm{CH}_{2} \mathrm{Cl}_{2}(10 \mathrm{~mL})$. The combined organic extracts were dried by filtration through cotton wool and evaporated in vacuo. Remaining sulfolane was removed by bulb to bulb distillation in vacuo of an oil pump at $120{ }^{\circ} \mathrm{C}$. The dark-green residue was purified by flash chromatography [silica gel (30 g), $\mathrm{CH}_{2} \mathrm{Cl}_{2} / \mathrm{MeOH} 97: 3,0.5 \% \mathrm{NEt}_{3}$ ] to yield a dark-blue-solid (2,3 mg, 49\% related to 9). For analytical purposes solids from different batches were combined and crystallized from $\mathrm{CHCl}_{3} /$-nentane.

Analytical data of $\mathbf{1 1 b}$ : $\mathrm{mp}>350{ }^{\circ} \mathrm{C}$ (decomposition). TLC (silica gel, $\mathrm{CH}_{2} \mathrm{Cl}_{2} / \mathrm{EtOAC}$ :1): $\mathrm{R}_{\mathrm{f}}{ }^{1}=0.73$. UV/Vis $\left[\mathrm{CHCl}_{3}\right.$ /petroleum ether, $\left.\lambda, \mathrm{nm},\left(\varepsilon, \mathrm{Lmol}^{-1} \mathrm{~cm}^{-1}\right)\right]: 398$ (100309), 502 (4687), 536 (3750), 573 (4687), 617 (30000). IR (solid, $\mathrm{NaCl}$, vmax, cm${ }^{-1}$ ): 3422, 3017, 2958, 2926, 2855, 1725, 1620, 1461, 1379, 1263, 1216, 1075, 948, 759, 668. $1 \mathrm{H} \mathrm{NMR} \mathrm{(360} \mathrm{MHz,} \mathrm{CDCl} 3+$ trace of $\mathrm{D}_{5}$ pyridine): $\delta_{\mathrm{H}} 1.84\left[6 \mathrm{H}, \mathrm{s}, 2 \mathrm{H}_{3} \mathrm{C}-\mathrm{C}(9)\right], 2.35[4 \mathrm{H}, \mathrm{m}$, $\left.\mathrm{H}_{2} \mathrm{C}(2), \mathrm{H}_{2} \mathrm{C}(4)\right], 3.15,3.28\left(12 \mathrm{H}, 2 \mathrm{~s}, \mathrm{H}_{3} \mathrm{C}-\mathrm{C}(19), \mathrm{H}_{3} \mathrm{C}-\mathrm{C}(20), \mathrm{H}_{3} \mathrm{C}-\mathrm{C}(14), \mathrm{H}_{3} \mathrm{C}-\mathrm{C}(15)\right], \quad 3.89$ [2H, m, H-C(5)], 3.99 $\left[2 \mathrm{H}, \mathrm{m}, \mathrm{H}_{2} \mathrm{C}(1)\right], 4.12\left(4 \mathrm{H}, \mathrm{m}, \mathrm{OCH}_{2} \mathrm{CH}_{2} \mathrm{O}\right), 4.36\left[2 \mathrm{H}, \mathrm{s}, \mathrm{H}_{2} \mathrm{C}(10)\right], 8.38[1 \mathrm{H}, \mathrm{s}, \mathrm{HC}(22)], 8.44[1 \mathrm{H}, \mathrm{s}, \mathrm{HC}(12)], 9.37$ [1H, s, HC(17)], $9.44[1 \mathrm{H}, \mathrm{s}, \mathrm{HC}(22)]$. Exact attribution of protons at $\mathrm{C}(22), \mathrm{C}(17), \mathrm{C}(12), \mathrm{C}(7)$ and at $\mathrm{C}(1)$ und $\mathrm{C}(5)$ was determined by a 2D-NOESY experiment. El-MS $\left(70 \mathrm{eV}, 200{ }^{\circ} \mathrm{C}\right): \mathrm{m} / \mathrm{z}(\%) 590\left(7,\left[\mathrm{M},{ }^{13} \mathrm{C}_{2},{ }^{68} \mathrm{Zn}\right]^{+}\right), 589$ $\left(19,\left[\mathrm{M},{ }^{13} \mathrm{C},{ }^{68} \mathrm{Zn}\right]^{+}\right), 588\left(34,\left[\mathrm{M},{ }^{68} \mathrm{Zn}\right]^{+}\right), 587\left(18,\left[\mathrm{M},{ }^{13} \mathrm{C}^{66} \mathrm{Zn}^{+}\right) 586\left(46,\left[\mathrm{M},{ }^{66} \mathrm{Zn}\right]^{+}\right), 585\left(22,\left[\mathrm{M},{ }^{13} \mathrm{C}{ }^{64} \mathrm{Zn}\right]^{+}\right), 584\right.$ $\left(73,\left[\mathrm{M},{ }^{64} \mathrm{Zn}\right]^{+}\right), 556\left(5,\left[\mathrm{M}-\mathrm{C}_{2} \mathrm{H}_{4}\right]^{+}\right), 543(5), 529(7), 528\left(8,\left[\mathrm{M}-\mathrm{C}_{2} \mathrm{O}_{2}\right]^{+}\right) .527(8), 526(7), 525(6), 472(6), 471$ (5), 470 (11), 468 (5), 457 (6), 455 (7). Isotope distribution of $\mathrm{M}^{+}$-Peaks (\%): Calcd 590 (4.3), 589 (15.9), 588 (45.6), 587 (31.3), 586 (64.5), 585 (38.5), 584 (100). Found 590 (10), 589 (25.6), 588 (47.1), 587 (25.2), 586 (62.6), 585 (30.6), 584 (100). HRMS: Calcd for $\left(\mathrm{C}_{33} \mathrm{H}_{36} \mathrm{~N}_{4} \mathrm{O}_{2}{ }^{64} \mathrm{Zn}^{+}\right)$584.21295. Found 584.21206.

Analytical of of 11a were not determined because 11a was only present as a minor component and due to the fact that in the next synthesis step a single product $\mathbf{1 2}$ is formed from 11a,b.

9,10-Dihydro-9,9,14,15,19,20-hexamethyl-24H,26H-cyclohepta[b]porphin-3-on (12). To chlorin ketal 11b together with 11a ( $2 \mathrm{mg}, 3.4 \mu \mathrm{mol})$ in $\mathrm{CH}_{3} \mathrm{CN}$ was added perchloric acid $(0.22 \mu \mathrm{L}, 70 \%)$ and water $(10 \mu \mathrm{L})$. The mixture was treated for $15 \mathrm{~min}$ at $\mathrm{rt}$ under an argon atmosphere in an ultrasonic bath. The reaction mixture was poured with $\mathrm{CH}_{2} \mathrm{Cl}_{2}$ into a separating funnel which contained aqueous, saturated $\mathrm{NaHCO}_{3}(10 \mathrm{~mL})$. After being exhaustively extracted with $\mathrm{CH}_{2} \mathrm{Cl}_{2}$, combined organic layers were dried by filtration through cotton wool and evaporated. The green solid was purified by flash chromatography [silica gel (10 g), $\mathrm{CH}_{2} \mathrm{Cl}_{2} / \mathrm{EtOAC}$ 
1\% $\mathrm{NEt}_{3}$ ]. Crystallization from $\mathrm{CHCl}_{3} / n$-pentane gave 12 as dark green microcrystals (1.6 mg, 97\%). For analytical purposes crystals from different batches were combined. $\mathrm{mp}>350{ }^{\circ} \mathrm{C}$ (decomposition). TLC (silica gel, $\mathrm{CH}_{2} \mathrm{Cl}_{2}$ /EtOAc 9:1): $\mathrm{R}_{\mathrm{f}}{ }^{1}=0.66$. UV/Vis $\left[\mathrm{CHCl}_{3} /\right.$ petroleum ether $\left.\lambda, \mathrm{nm}\left(\varepsilon, \mathrm{Lmol}^{-1} \mathrm{~cm}^{-1}\right)\right]: 390$ (190945), 495 (11811), 593 (3937), 646 (61024). IR (solid, KBr, vmax, cm ${ }^{-1}$ ): 3340 (NH), 3020, 2918, 2850, 1702 (C=O), 1615, $1559,1522,1457,1163,1047,906.1 \mathrm{H} \mathrm{NMR}\left(360 \mathrm{MHz}, \mathrm{CDCl}_{3}\right): \delta_{\mathrm{H}}-2.51(2 \mathrm{H}$, broad s, $2 \mathrm{NH}), 2.06[6 \mathrm{H}, \mathrm{s}, 2 \mathrm{Me}-$ $\mathrm{C}(9)], 3.37\left[2 \mathrm{H}, \mathrm{m}, \mathrm{H}_{2} \mathrm{C}(2)\right], 3.42\left[2 \mathrm{H}, \mathrm{m}, \mathrm{H}_{2} \mathrm{C}(4)\right], 3.39,3,49(12 \mathrm{H}, 2 \mathrm{~s}, \mathrm{Me}-\mathrm{C}(14), \mathrm{Me}-\mathrm{C}(15), \mathrm{Me}-\mathrm{C}(19), \mathrm{Me}-$ $\mathrm{C}(20)], 4.35\left[2 \mathrm{H}, \mathrm{m}, \mathrm{H}_{2} \mathrm{C}(5)\right], 4.45\left[2 \mathrm{H}, \mathrm{m}, \mathrm{H}_{2} \mathrm{C}(1)\right], 4.63\left(2 \mathrm{H}, \mathrm{s}, \mathrm{H}_{2} \mathrm{C}(10)\right], 8.75[1 \mathrm{H}, \mathrm{s}, \mathrm{HC}(7)], 8.91[1 \mathrm{H}, \mathrm{s}$, $\mathrm{HC}(12)], 9.64[1 \mathrm{H}, \mathrm{s}, \mathrm{HC}(17)], 9.69[1 \mathrm{H}, \mathrm{s}, \mathrm{HC}(22)]$. Exact attribution of protons was determined by 2D-NOESY experiments. El-MS $\left(70 \mathrm{eV}, 200{ }^{\circ} \mathrm{C}\right): \mathrm{m} / \mathrm{z}(\%) 480\left(10,\left[\mathrm{M},{ }^{13} \mathrm{C}_{2}\right]^{+}\right), 479\left(39,\left[\mathrm{M},{ }^{13} \mathrm{C}\right]^{+}\right), 478\left(100, \mathrm{M}^{+}\right) . \mathrm{HRMS}^{\mathrm{C}} \mathrm{Calcd}$ for $\left(\mathrm{C}_{31} \mathrm{H}_{34} \mathrm{~N}_{4} \mathrm{O}\right)$ 478.27325. Found 478.27265. Anal. calcd for $\left(\mathrm{C}_{31} \mathrm{H}_{34} \mathrm{~N}_{4} \mathrm{O} \times 0.24 \mathrm{CHCl}_{3}\right)$ 507,33: $\mathrm{C}, 73.39 ; \mathrm{H}$, 6.76, N, 11.04. Found C, 73.25; H, 6.55; N, 12,30.

10,11-Dihydro-10,10,15,16,20,21-hexamethyl-25H,27H-3-azacycloocta[b]porphin-4-on (13a) and 10,11,dihydro-10,10,15,16,20,21-25H,27H-4-azabicycloocta[b]porphin-3-on (13b). To a solution of chlorin ketone (12) $(1 \mathrm{mg}, 2.1 \mu \mathrm{mol})$ in $\mathrm{CH}_{2} \mathrm{Cl}_{2}(1 \mathrm{~mL})$ was added a solution of $O$-mesityl sulfonyl hydroxylamine $(0.8 \mathrm{mg}, 3.7$ $\mu \mathrm{mol})$ in $\mathrm{CH}_{2} \mathrm{Cl}_{2}(1 \mathrm{~mL})$ at $0{ }^{\circ} \mathrm{C}$ under an argon atmosphere. After being treated in an ultrasonic bath for $30 \mathrm{~min}$ at $\mathrm{rt}$, the solvent was completely evaporated. The residue was re-dissolved in benzene $(1 \mathrm{~mL})$ under treatment in an ultrasonic bath and a suspension of Alox Super I basic in $\mathrm{MeOH}(0.1 \mathrm{~mL})$ was added. After being stirred for $3 \mathrm{~h}$ at $\mathrm{rt}$, the suspension was filtered through a glass frit and the solvent evaporated. The residue was purified by flash chromatography [silica gel (ca. $5 \mathrm{~g}$ ), $\mathrm{CH}_{2} \mathrm{Cl}_{2} / \mathrm{EtOAc} 9: 1,1 \% \mathrm{NEt}_{3}$ ]. After evaporation of eluent, the mixture of constitutionally isomeric lactams $\mathbf{1 3 a , b}$ was obtained as a dark green solid (0.8 $\mathrm{mg}, 77 \%)$. For analytical purposes samples from different batches were combined and crystallized from $\mathrm{CHCl}_{3} / n$-pentane to give microcrystals of $13 a, b . \mathrm{mp}>350{ }^{\circ} \mathrm{C}$.

TLC (silica gel, $\mathrm{CH}_{2} \mathrm{Cl}_{2} /$ EtOAc 9:1): $\mathrm{R}_{\mathrm{f}}$ 0.81. HPLC (Nucleosil Chiral 2, $n$-heptane/dioxane 70:30, $\left.1 \mathrm{~mL} / \mathrm{min}\right): \mathrm{t}_{\mathrm{R}}{ }^{2}=$ 16 min (peak integral 1): 13b; $t_{R}{ }^{1}=17.5$ min (peak integral 1.5): 13a.

UV/Vis $\left[\mathrm{CHCl}_{3}, \lambda, \mathrm{nm}\left(\varepsilon, \mathrm{Lmol}^{-1} \mathrm{~cm}^{-1}\right)\right]: 391$ (126903), 495 (15663), 590 (2708, 616 (2655), 647(45133). IR (solid, $\left.\mathrm{KBr}, \mathrm{vmax}, \mathrm{cm}^{-1}\right)$ : 3436, 3020, 2924, 2853, 1732, 1694, 1653, 1615, 1520, 1455, 1367, 1262, 1192, 1038, 817, 725, 663. ${ }^{1} \mathrm{H} N M R\left(360 \mathrm{MHz}, \mathrm{CDCl}_{3}\right)$ for 13a: $\delta_{\mathrm{H}} 2.1[6 \mathrm{H}, \mathrm{s}, 2 \mathrm{MeC}(10)], 3.44,3.54$ [12H, m, $\mathrm{MeC}(15), \operatorname{MeC}(16)$, $\mathrm{MeC}(20), \mathrm{MeC}(21)], 3.5\left[2 \mathrm{H}, \mathrm{m}, \mathrm{H}_{2} \mathrm{C}(5)\right], 3.53\left[2 \mathrm{H}, \mathrm{m} \mathrm{H}_{2} \mathrm{C}(2)\right], 4.45\left[2 \mathrm{H}, \mathrm{m}, \mathrm{H}_{2} \mathrm{C}(6)\right], 4.57\left[2 \mathrm{H}, \mathrm{m}, \mathrm{H}_{2} \mathrm{C}(1)\right], 4.68$ [2H, m, $\left.\mathrm{H}_{2} \mathrm{C}(11)\right], 5.89[1 \mathrm{H}$, broad s, $\mathrm{HN}(3)], 8.75[1 \mathrm{H}, \mathrm{s}, \mathrm{HC}(8)], 8.93[1 \mathrm{H}, \mathrm{s}, \mathrm{HC}(13)], 9.67[1 \mathrm{H}, \mathrm{s}, \mathrm{HC}(18)], 9.74$ $[1 \mathrm{H}, \mathrm{s}, \mathrm{HC}(23)]$. For 13b: $\delta_{\mathrm{H}}[6 \mathrm{H}, \mathrm{s}, 2 \mathrm{MeC}(10)], 3.44,3.54[12 \mathrm{H}, \mathrm{m}, \mathrm{MeC}(15), \mathrm{MeC}(16), \mathrm{MeC}(20), \mathrm{MeC}(21)], 3.45$ $\left[2 \mathrm{H}, \mathrm{m} \mathrm{H}_{2} \mathrm{C}(5)\right], 3.46\left[2 \mathrm{H}, \mathrm{m}, \mathrm{H}_{2} \mathrm{C}(2)\right], 4.49\left[2 \mathrm{H}, \mathrm{m}, \mathrm{H}_{2} \mathrm{C}(6)\right], 4.55\left[2 \mathrm{H}, \mathrm{m}, \mathrm{H}_{2} \mathrm{C}(1)\right], 4.68\left[2 \mathrm{H}, \mathrm{m}, \mathrm{H}_{2} \mathrm{C}(11)\right], 5.37$ [1H, broad s, $\mathrm{HN}(4)], 8.83[1 \mathrm{H}, \mathrm{s}, \mathrm{HC}(8)], 8.93[1 \mathrm{H}, \mathrm{s}, \mathrm{HC}(13)], 9.67[2 \mathrm{H}, \mathrm{s}, \mathrm{HC}(18), \mathrm{HC}(23)]$. Tentative attribution of protons of constitutional isomers $13 \mathrm{a}$ and $13 \mathrm{~b}$ was achieved by 2D-NOESY and 2D-NOESY-HH-COSY experiments. Attribution and designation of constitutions was facilitated by the fact that the constitutional isomers $\mathbf{1 3 a}$ and $\mathbf{1 3 b}$ were formed and are present in the NMR sample in a 1.5:1 ratio. DCl-MS (negative, $\left.\mathrm{NH}_{3} / \mathrm{NH}_{4}{ }^{+}, \mathrm{mA} / \mathrm{sec}\right): \mathrm{m} / \mathrm{z}$ (\%) \%48 (6), $547(8), 494\left(15\left[\mathrm{M}^{13}{ }^{13} \mathrm{C}\right]^{-}\right), 493\left(60, \mathrm{M}^{-}\right), 492\left(10[\mathrm{M}-\mathrm{H}]^{-}\right), 491(10), 477$ (14), 476 (15), 475 (40), 474 (5), 473 (17), 460 (14), 391 (5), 390 (14), 199 (21), 198 (11), 168 (5), 166 (11), 152 (6), 151 (18), 150 (25), 148 (13), 147 (23), 136 (5), 125 (11), 121 (10), 119 (25), 83 (15).

HRMS: Calcd for $\left(\mathrm{C}_{31} \mathrm{H}_{35} \mathrm{~N}_{5} \mathrm{O}\right)$ 493.28415. Found 493.28470. 


\section{Acknowledgements}

We thank Dr. P. Schulze, Mrs. I. Erxleben, Dipl.-Ing. J. Stelten (Institute of Organic and Analytical Chemistry, Laboratory Prof. Dr. D. Leibfritz, University of Bremen) for mass spectrometry and NMR spectroscopy measurements. In particular we are indebted Mrs. A. Lincke for performing HPLC separations. This work was supported by Deutsche Forschungsgemeinschaft.

\section{References}

1. Kirmaier, C.; Holten, D. In Photosynthetic Reaction Center; Deisenhofer, J.; Michel, H.; Norris, J. R., Eds.; Academic Press: San Diego, 1993; pp 49-70.

https://doi.org/10.1016/B978-0-12-208662-5.50008-6

2. Deisenhofer, J.; Michel, H. In Photosynthetic Reaction Center; Deisenhofer, J.; Michel, H.; Norris, J. R., Eds.; Academic Press: San Diego, 1993; pp 541-558.

https://doi.org/10.1016/B978-0-12-208662-5.50022-0

3. Parson, W. W. New Compr. Biochem. 1987, 15, 43-61. https://doi.org/10.1016/S0167-7306(08)60134-9

4. Deisenhofer, J.; Epp, O.; Sinning, I.; Michel, H. J. Mol. Biol. 1995, 246, 429-57. https://doi.org/10.1006/jmbi.1994.0097

5. Huber, R. Angew. Chem., Int. Ed. Engl. 1989, 28, 848-869. https://doi.org/10.1002/anie.198908481

6. Deisenhofer, J.; Michel, H. Angew. Chem., Int. Ed. Engl. 1989, 28, 829-847. https://doi.org/10.1002/anie.198908293

7. Fromme, P.; Jordan, P.; Krauss, N. Biochim. Biophys. Acta, Bioenerg. 2001, 1507, 5-31. https://doi.org/10.1016/S0005-2728(01)00195-5

8. Gust, D.; Moore, T. A. In Topics in Current Chem.; Mattay, J., Ed.; Springer: Berlin, 1991; Vol. 159, pp. 103151.

9. Wasielewski, M. R. Chem. Rev. 1992, 92, 435-461. https://doi.org/10.1021/cr00011a005

10. Kurreck, H ; Huber, M. Angew. Chem., Int. Ed. Engl. 1995, 34, 849-866. https://doi.org/10.1002/anie.199508491

11. Gust, D.; Moore, T. A. In Handbook of Porphyrin Chemistry; Kadish, K. M.; Smith, K. M.; Guilard, R. Eds.; Academic Press : San Diego, 2000; Vol. 8, 153-190.

12. Tauber, A. Y.; Kostiainen, R. K.; Hynninen, P. H. Tetrahedron 1994, 50, 4723-32. https://doi.org/10.1016/S0040-4020(01)85011-0

13. Borovkov, V. V.; Gribkov, A. A.; Kozyrev, A. N.; Brandis, A. S.; Ishida, A.; Sakata, Y. Bull. Chem. Soc. Jpn. 1992, 65, 1533-7. https://doi.org/10.1246/bcsj.65.1533

14. Maruyama, K.; Yamada, H.; Osuka, A. Chem. Lett. 1989, 18, 833-836. https://doi.org/10.1246/cl.1989.833

15. Tauber, A. Y.; Helaja, J.; Kilpelainen, I.; Hynninen, P. H. Acta Chem. Scand. 1997, 51, 88-93. https://doi.org/10.3891/acta.chem.scand.51-0088 
16. Abel, Y.; Montforts, F.-P. Tetrahedron Lett. 1997, 38, 1745-1748. https://doi.org/10.1016/S0040-4039(97)00186-X

17. Montforts, F.-P.; Gerlach, B.; Hoeper, F. Chem. Rev. 1994, 94, 327-347. https://doi.org/10.1021/cr00026a003

18. Montforts, F.-P.; Glasenapp-Breiling, M. In Progress in the Chemistry of Organic Natural Products; Herz, W.; Falk, H.; Kirby, G. W., Eds.; Springer: Wien, New York, 2002 ; Vol. 84, 1-51.

19. Lindsey, J. S. Chem. Rev. 2015, 115, 6534-6620. https://doi.org/10.1021/acs.chemrev.5b00065

20. Taniguchi, M.; Lindsey, J. S. Chem. Rev. 2017, 117, 344-535. https://doi.org/10.1021/acs.chemrev.5b00696

21. Mass, O.; Taniguchi, M.; Patszek, M.; Springer, J. W.; Faries, K. M.; Diers, J. R.; Bocian, D. F.; Holten, D.; Lindsey, J. S. New J. Chem. 2011, 35, 76-88. https://doi.org/10.1039/CONJ00652A

22. Montforts, F.-P. Angew. Chem., Int. Ed. Engl. 1981, 20, 778-779. https://doi.org/10.1002/anie.198107781

23. Montforts, F.-P.; Schwartz, U. M. Liebigs Ann. Chem. 1985, 1228-53. https://doi.org/10.1002/ilac.198519850614

24. Montforts, F.-P.; Kutzki, O. Angew. Chem. Int. Ed. 2000, 39, 599-601. https://doi.org/10.1002/(SICI)1521-3773(20000204)39:3<599::AID-ANIE599>3.0.CO;2-X

25. Koenekamp, T.; Ruiz, A.; Duwenhorst, J.; Schmidt, W.; Borrmann, T.; Stohrer, W.; Montforts, F.-P. Chem. Eur. J. 2007, 13, 6595-6604. https://doi.org/10.1002/chem.200700222

26. Koenekamp, T.; Borrmann, T.; Montforts, F.-P. J. Porphyrins Phthalocyanines 2009, 13, 166-170. https://doi.org/10.1142/S1088424609000127

27. Thanh, N. T. V.; Koenekamp, T.; Hanke, D.; Loewer, F.; Borrmann, T.; Montforts, F.-P. J. Porphyrins Phthalocyanines 2012, 16, 626-632.

https://doi.org/10.1142/S1088424612500666

28. Bertran, J. C.; Montforts, F.-P. Eur. J. Org. Chem. 2017, 1608-1617. https://doi.org/10.1002/ejoc.201601504

29. Abel, Y.; Haake, E.; Haake, G.; Schmidt, W.; Struve, D.; Walter, A.; Montforts, F.-P. Helv. Chim. Acta 1998, 81, 1978-1996. https://doi.org/10.1002/(SICI)1522-2675(19981111)81:11<1978::AID-HLCA1978>3.0.CO;2-T

30. Haake, G.; Struve, D.; Montforts, F.-P. Tetrahedron Lett. 1994, 35, 9703-9704. https://doi.org/10.1016/0040-4039(94)88364-5

31. Tamura, Y.; Fujiwara, H.; Sumoto, K.; Ikeda, M.; Kita, Y. Synthesis 1973, 215-16.

32. Minamikawa, J.; Ikeda, M.; Tamura, Y. Synthesis 1977, 1-17. 